\title{
Method for the simulation and reconstruction of 3D particle size distributions in metal structures
}

\author{
Tetiana Danilenko* \\ State University of Infrastructure and Technologies, Ivan Ohienko str, 19, 03049 Kyiv, Ukraine
}

\begin{abstract}
The stereological method for studying grain structures is presented, it determines the interrelation between the size distributions of three-dimensional grains and their planar and linear sections. Method is applicable for both reconstruction and simulation of the 3D structures. The polyhedral shape of the grains is taken into account by means of the shape factors established for them. An original method to determine the distribution of plane sections from the certain grain-polyhedron to calculate the shape factors is presented. This method can be used to describe the shape of different structural components in many materials: steels, cast irons, concretes, rocks, ceramics, open-porosity materials, biofilms, foams, etc. The stereological method is also applicable to structures with spherical particles, for which the shape factors are equal to unity. The method makes it possible to create the standards of plane sections of grain structures and other structures with a previously known size distribution of $3 \mathrm{D}$ grains or particles.
\end{abstract}

\section{Introduction}

Material properties, including mechanical ones, depend on quantitative characteristics of their 3D structural components such as grains, voids, inclusions having a range of sizes and shapes. Examples of such materials are metals, concretes, rocks, ceramics, open-porosity materials, biofilms, foams, etc. Size distribution of their structural components is an important characteristic of materials [1-3] and it can be determined by reconstruction from a series of parallel sections planes [4-6] or by stereological methods that apply mathematical relations between 2D and 3D geometry [7, 8]. Stereological methods have an advantage over serial sections methods, because they are not so laborious and do not require such a significant material destruction as the parallel sections techniques.

The best known stereological methods for determining the distribution of spherical particles are described in $[9,10]$. However, many structural particles have a non-spherical shape, for example in metals, ceramics, concretes and others. The particles can have the shape that differs significantly from sphere, e.g. cubic $\gamma^{\prime}$ phase particles in nickel-based superalloys [11] or cement particles in concrete [12]. The study of the distributions of nonspherical particles, including grain-polyhedrons, is trying to apply stereological methods developed for spheres, but the difference between their shapes initially makes an obvious

* Corresponding author: danilenko.stereo.7@,gmail.com 
error in the result. Thus, the key problem of stereology in determining the $3 \mathrm{D}$ particle size distribution is the recognition of the shape of these particles [13].

The purpose of this work is to present the method of stereological reconstruction and simulation three diameter size distribution (3DSD) that is applicable for the grain polycrystalline metal structures and structures with spherical particles.

\section{Materials and method}

Initially, the method was developed for a spherical particle system (SPS) with a diameter distribution $P\left(D_{k}\right)$ in a scale of $n$ discrete intervals. The main points of this method for the SPS are presented in the works $[14,15]$. When the SPS is dissected by a plane, a system of plane sections with a diameter distribution $P_{k}(d)$ is obtained. Drawing on the resulting plane section of the lines gives a system of chords with the distribution of their lengths $P_{k}(l)$.

For particles of non-spherical shape it is proposed to use the shape factors of these particles [16]. If the shape of the particles is not uniform, then the shape factors are determined for a particle of the average shape, as will be done below for grain-polyhedrons. Shape factors $\alpha_{i}$ and $\beta_{i}$ are intended to account for each size interval the differences in the distributions of $P_{k}(d)$ from the sphere and particle, and the differences in the distributions $P_{k}(l)$ from the circle and plane section of the particle, in accordance with that the shape factors need to be found from the equations:

$$
\begin{gathered}
\alpha_{i}=P_{k}(d)_{\text {particle }} / P_{k}(d)_{\text {sphere }} \\
\beta_{i}=P_{k}(l)_{\text {particle }} / P_{k}(l)_{\text {circle }}
\end{gathered}
$$

The resulting equations establishing the relation between the relative frequencies $P_{k}^{\prime}(d)$ and $P^{\prime}\left(D_{k}\right) ; P_{k}^{\prime}(l)$ and $P_{k}^{\prime}(d)$ are:

$$
\begin{aligned}
& P_{k}^{\prime}(d)=\alpha_{a} P^{\prime}\left(D_{k}\right) \sqrt{D_{k}^{2}-d_{k-1}^{2}}+\alpha_{b} P^{\prime}\left(D_{k+1}\right)\left(\sqrt{D_{k+1}^{2}-d_{k-1}^{2}}-\sqrt{D_{k+1}^{2}-d_{k}^{2}}\right)+\ldots+\alpha_{n} P^{\prime}\left(D_{n}\right)\left(\sqrt{D_{n}^{2}-d_{k-1}^{2}}-\sqrt{D_{n}^{2}-d_{k}^{2}}\right) \\
& P_{k}^{\prime}(l)=\beta_{a} P^{\prime}{ }_{k}(d) \sqrt{d_{k}^{2}-l_{k-1}^{2}}+\beta_{b} P^{\prime}{ }_{k+1}(d)\left(\sqrt{d_{k+1}^{2}-l_{k-1}^{2}}-\sqrt{d_{k+1}^{2}-l_{k}^{2}}\right)+\ldots+\beta_{n} P^{\prime}{ }_{n}(d)\left(\sqrt{d_{n}^{2}-l_{k-1}^{2}}-\sqrt{d_{n}^{2}-l_{k}^{2}}\right)
\end{aligned}
$$

For the SPS shape factors are equal to unity. The obtained values of $P_{k}^{\prime}(d)$ and $P_{k}^{\prime}(l)$ should be normalized in accordance with equations:

$$
\begin{aligned}
& P_{k}(d)=P_{k}{ }^{\prime}(d) / \sum_{k=1}^{n} P_{k}{ }^{\prime}(d) \\
& P_{k}(l)=P_{k}{ }^{\prime}(l) / \sum_{k=1}^{n} P_{k}{ }^{\prime}(l)
\end{aligned}
$$

The presented equations (3) and (4) allow to simulate different distributions $P\left(D_{k}\right)$ and calculate the corresponding distributions $P_{k}(d)$ and $P_{k}(l)$ and thus study the relation of the structure in volume and at its plane section. This is one of the important application of the method. The proposed simulation method can be widely used for the study of structures with spherical particles, of various surfaces that are formed by systems of hard spheres or which can be modelled by SPS. Examples of such systems are liquid-gas interfaces, the interfaces between fluid and porous substrate, surface cracks in concrete, where the hard spheres denote the aggregate particles, porosity profiles, where the pores are considered as spheres. Thus, the created method makes it possible to replace by simple calculation complex procedures of constructing models of spherical particle structures and their dissection by planes, as discussed in $[3,6,17,18]$. 
For the reconstruction of 3DSD by this method two types of initial information can be used: $P_{k}(d)$ or $P_{k}(l)$, determined experimentally. In the first case we need to compile a system of equations (3), and solve it relatively unknowns $P^{\prime}\left(D_{k}\right)$, then the results are normalized in accordance with the equation:

$$
P\left(D_{k}\right)=P^{\prime}\left(D_{k}\right) / \sum_{k=1}^{n} P^{\prime}\left(D_{k}\right)
$$

If the initial information is $P_{k}(l)$, then first we need to compile a system of equations (4) and solve it relatively unknowns $P_{k}^{\prime}(d)$, which are then normalized. Then compile the system of equations (3) using the found $P_{k}(d)$ as knowns and solve it relatively unknowns $P^{\prime}\left(D_{k}\right)$, which then are also normalized.

\section{Results and discussion}

The presented method was applied to analysis of the grain recrystallized austenite structure. The distributions $P_{k}(d)_{\text {grain }}$ and $P_{k}(l)_{\text {grain }}$ were determined as the average for a statistically sufficient group of grains, i.e. for the 3D grain of average shape. The definition of $P_{k}(l)_{\text {grain }}$, necessary to determine the shape factors $\beta_{i}$, was not difficult, as will be discussed later. And to establish the $P_{k}(d)_{\text {grain }}$ from the 3D grain of average shape, necessary for calculating the shape factors $\alpha_{i}$, the original method is proposed. According to the well-known principle of Cavalieri-Aker, the proportion of the structural component in the volume of the material is equal to its proportion on a plane section. In accordance to this principle it was assumed that the distribution of plane section diameters $P_{k}(d)$ from a certain 3D grain is close to the distribution of chords $P_{k}(l)$ at its maximum plane section, if the plane sections of $3 \mathrm{D}$ grain from each dimensional group are treated as separate structural components. The interrelation between the size of plane sections of 3D grain and the length of the chords is shown in Fig. 1. The plane sections Q, M and P, intersecting with the maximum for this $3 \mathrm{D}$ grain plane section $\mathrm{G}$, produce on it chords, respectively, $E F, C D$ and $A B$. The larger the plane section area, the longer the chord it gives on the maximum section $G$. That is, it should be expected that if the proportion of plane sections from the 3D grain in the $k$-th size interval is $P_{k}(d)$, then at the maximum plane section of this grain the proportion of chords in the $k$-th size chord interval $P_{k}(l)$ will be approximately equal to $P_{k}(d)$.

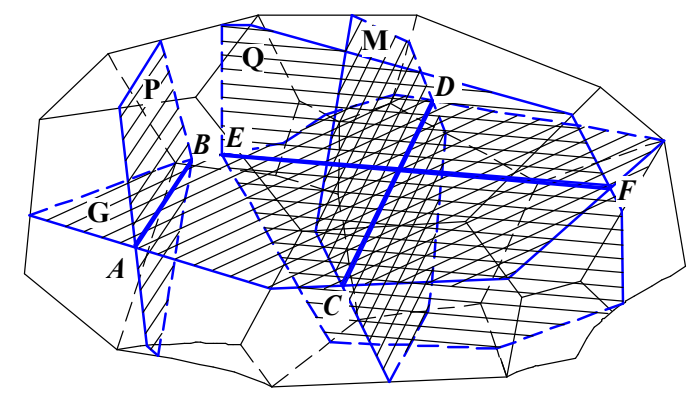

Fig. 1. Scheme of dissection of the metal grain model by planes (shaded) and obtaining chords EF, $\mathrm{CD}, \mathrm{AB}$ as a result of intersections of planes $\mathrm{Q}, \mathrm{M}, \mathrm{P}$ with the maximum grain section $\mathrm{G}$

The assumed assumption is verified for five polyhedrons, which can be considered as models of grains: pentagonal dodecahedron, tetrakaidekahedron, cubic octahedron, rhombic dodecahedron and truncated icosahedron that were studied in the works of other authors. As $P_{k}(d)$ used the data obtained by Adrian [19] for pentagonal dodecahedron, tetrakaidekahedron and cubic octahedron; by Hull and Houk [20] for rhombic 
dodecahedron and by Matsuura and Itoh [21] for truncated icosahedron. In [19] it is presented the distributions of the plane section perimeters for polyhedrons, which were adopted in this paper for the diameter distributions of the equal circles, based on the relation of the circle perimeter and diameter. In [20] and [21] done the distribution $P_{k}(d)$. To present the results of these works in the required size scale, $P_{k}(d)$ were determined as the area under the graphs for each size interval, as shown for $(0.6-0.7)$ relative size interval for tetrakaidekahedron [19] in Fig. 2. This method was used to determine the distribution $P_{k}(d)$ for these five polyhedrons in scales of 10, 15 and 20 intervals.

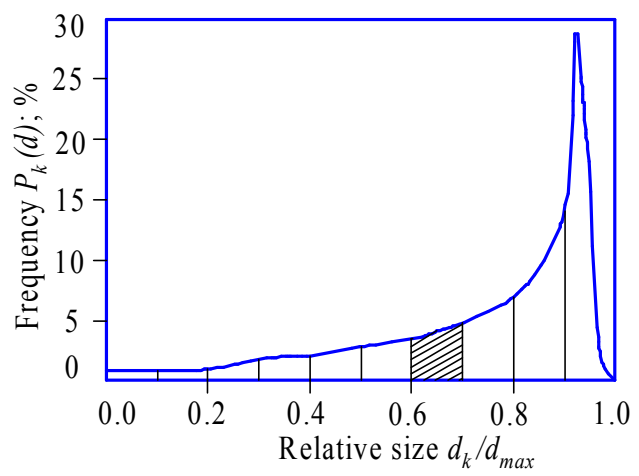

Fig. 2. The scheme for determining the area under the graph (taken from Adrian (1990) for the relative size interval $(0.6-0.7)$ for tetrakaidekahedron

The distributions $P_{k}(l)$ were determined experimentally on the maximum plane sections of these polyhedrons using the computer program «Compass-3D», in which these plane sections were created, then the secant lines were applied in 32 directions at an equal distance between the lines in the same direction, which ensured the statistical uniformity of the secant lines. The number of chords for one plane section was $2500-3000$ pieces, which, as shown by the additional studies, is sufficient. The results presented in Fig. 3 demonstrate the validity of the assumption that the distribution $P_{k}(d)$ of polyhedron is close to $P_{k}(l)$ obtained at its maximum plane section. These distributions are especially close for a size scale of 10 intervals (Fig. $3 f, g, h, k, m$ ). For scales of 15 and 20 intervals, there are differences in the values of $P_{k}(d)$ and $P_{k}(l)$ in the two or three last size intervals. However, in general, these distributions are close enough, that gave grounds to applying this solution also for obtain $P_{k}(d)_{\text {grain }}$ of austenite grains, for which it is to be expected that the indicated differences in the distributions in scales of 15 and 20 intervals will be partially levelled due to the greater diversity of their shapes in comparison with the investigated polyhedrons.

To determine the shape factors of austenitic grains as outlined above, the shape of about 3000 plane sections of $3 \mathrm{D}$ grains was visually analysed and 20 maximum sections of the most representative shape were selected. Since these plane sections were of maximum sizes observed in the structure, they were considered to be the maximum plane sections of the grains from which they were obtained. On each of these plane sections, in accordance with the procedure described above for polyhedrons, $P_{k}(l)$ was determined using the computer program «Compass-3D» and took it as $P_{k}(d)$ from these 3D grains, and then determined the average value of $P_{k}(d)$ for each size interval for 20 grains. The obtained $P_{k}(d)$ adopted for $P_{k}(d)_{\text {grain }}$ from the 3D grain of the average shape. The number of grains chosen for analysis was considered sufficient, because $P_{k}(d)_{\text {grain }}$, established for two groups of 10 analysed grains, had insignificant differences between them.

To establish the $P_{k}(l)_{\text {grain }}$ the same 20 maximum grain sections were used, on which $P_{k}(d)_{\text {grain }}$ was found, and an additional 20 plane sections of the non-maximal sizes, since they should also represent the average distribution $P_{k}(l)_{\text {grain }}$. 


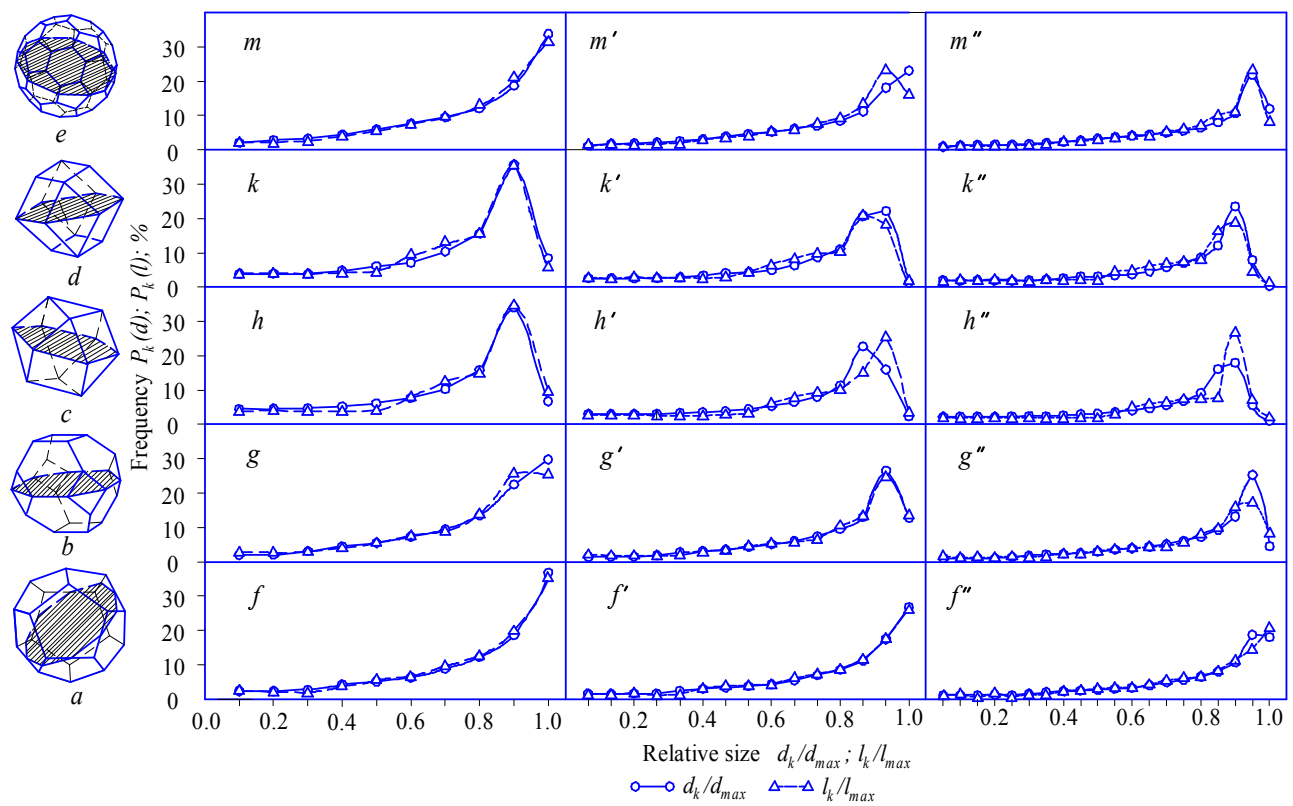

Fig. 3. Models of polyhedrons: pentagonal dodecahedron (a), tetrakaidekahedron (b), cubic octahedron (c), rhombic dodecahedron (d), truncated icosahedron (e) and their maximum plane sections (shaded) and distributions $P_{k}(d)$ from polyhedrons respectively $\left(f, f^{\prime}, f^{\prime \prime}, g, g^{\prime}, g^{\prime \prime}, k, k^{\prime}, k^{\prime \prime}\right.$ taken from Adrian (1990); $h, h^{\prime}, h^{\prime \prime}$ taken from Hull and Houk (1953); m, m', m" taken from Matsuura and Itoh (1991) and distributions $P_{k}(l)$ obtained experimentally from the maximum plane section of polyhedrons

$P_{k}(d)_{\text {sphere }}$ and $P_{k}(l)_{\text {circle }}$ were calculated for a sphere with a relative diameter of 1 conventional unit by the equation:

$$
P_{k}(d)_{\text {sphere }}=P_{k}(l)_{\text {circle }}=\sqrt{1-d_{k-1}^{2}}-\sqrt{1-d_{k}^{2}}
$$

Further, according to equations (1) and (2), the shape factors $\alpha_{i}$ and $\beta_{i}$ were calculated for uniform scales of 10 and 20 intervals, taking into account that from the plane section of the $k$-th size interval chords are obtained only in the size intervals from k-th to 1-th. A computer program has been developed for all calculations. The graphs of the distributions $P_{k}(d)_{\text {grain }}$ and $P_{k}(l)_{\text {grain }}$ obtained and $P_{k}(d)_{\text {sphere }}\left(P_{k}(l)_{\text {circle }}\right)$ calculated according with equations (8) are shown in Fig. 4, where the proximity of the distributions of $P_{k}(d)_{\text {grain }}$ and $P_{k}(l)_{\text {grain }}$ and the distinct difference between these distributions and $P_{k}(d)_{\text {sphere }}$ and $P_{k}(l)_{\text {circle }}$ are seen. The obtained result indicates that the stereological reconstruction of the $3 \mathrm{D}$ grain structure by the methods created for spherical particles can lead to erroneous results and conclusions. The results obtained agree with the data of [20] where was showed, that the maximum of distribution $P(d)$ for the regular polyhedrons is between 0.85 and $0.95 d / d_{\max }$, and concluded that it should be expected that the maximum frequency $P(d)$ for the average grain will not be as high and narrow as for the regular polyhedrons and an increase in the values of $P(d)$ will be observed at lower value of $d / d_{\max }$. These assumptions are in good agreement with the results presented in Fig. 4. The resulting distribution $P_{k}(d)_{\text {grain }}$ has a maximum in the range $0.8-0.9$ relative size $d / d_{\max }$, i.e. is shifted to smaller values, and is wider, not so high, and has large values in the range of small $d / d_{\max }$, for example, $P_{k}(d)$ is 10.3 for grains and $(5.2-6.2)$ for polyhedrons at $d / d_{\max }$ equal to 0.6 .

In the work [22] 3D simulation of grain structures and SPS in which grains and particles had different size distributions was done. The simulation of $P\left(D_{k}\right)$ showed significant 
differences of the corresponding distributions $P_{k}(d)$ and $P_{k}(l)$ for grain-polyhedrons and spheres, which indicates that the assumption that the grain-polyhedrons can be considered as spheres in stereological studies is incorrect. In addition, the distributions of sections have differences from the distributions of the 3D particles, the same applies to the distribution parameters (mean diameter, coefficient of variation, etc.).

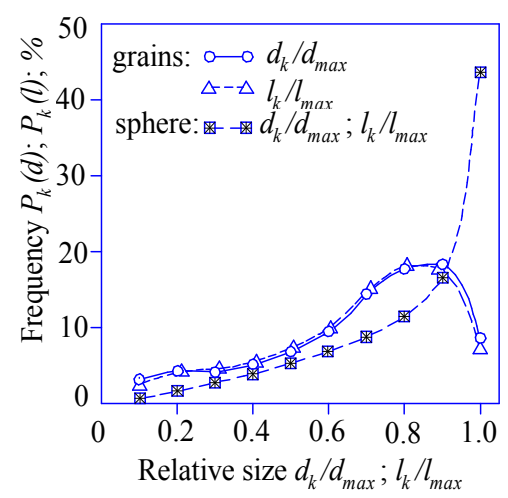

Fig. 4. The distributions $P_{k}(d)_{\text {grain }}$ and $P_{k}(l)_{\text {grain }}$ from the grain of average shape and $P_{k}(d)_{\text {sphere }}$ and $P_{k}(l)_{\text {circle }}$ from the sphere

\section{Creation of the version of the structure standard}

The important area of application of the proposed method is the possibility to create the plane section standards of the 3D structures with a known 3-D particles distribution [16]. In this paper a standard version of the plane section of the $3 \mathrm{D}$ grain structures was created in the form of the distribution $P_{k}(d)$ corresponding to the quantitative characteristics of the grain size index $G 6$ of ISO 643:2012 [23], but with a previously known distribution of 3D grains $P\left(D_{k}\right)$. To do this, first the existing standard $G 6$ [23] was analysed in the «Compass $3 \mathrm{D} »$ program, for this its scheme was created, the areas of plane grains were measured and the diameter distribution $P_{k}(d)$ of the equal circles was determined.

Then the distribution $P\left(D_{k}\right)$ was chosen, which gave a plane section distribution $P_{k}(d)$ with the quantitative characteristics corresponded to the distribution $P_{k}(d)_{I S O} 643$ of existing standard $G 6$. The distributions $P\left(D_{k}\right)$ of the created version of the standard $G 6$ and the corresponding $P_{k}(d)$ and $P_{k}(l)$, as well as $P_{k}(d)_{I S O} 643$ of the existing standard $G 6$ are presented in Fig. 5, and the parameters of all distributions are in Table.

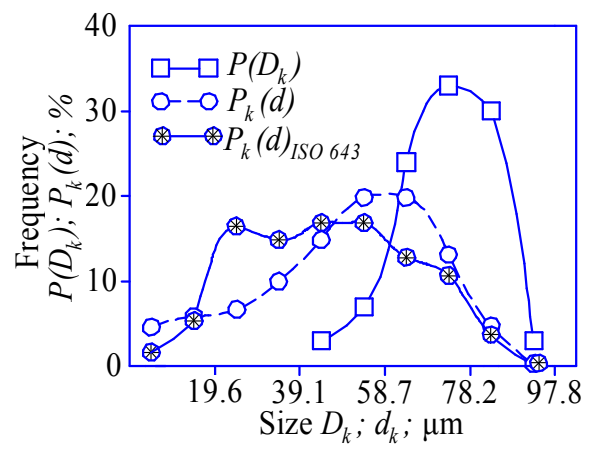

Fig. 5. Distribution of the plane section diameters $P_{k}(d)_{I S O} 643$ of the standard $G 6$ (ISO 643:2012) and the distribution of $3 \mathrm{D}$ grains $P\left(D_{k}\right)$ taken to create a variant of the plane section $P_{k}(d)$ close to the standard G6 
These results showed that the plane section of the 3D grain structure which corresponds to the grain size index $G 6$ with the mean diameter of the plane grains $d=44.2 \mu \mathrm{m}$ gives a $3 \mathrm{D}$ grain structure with a mean diameter $D=72.3 \mu \mathrm{m}$, i.e. much larger than observed on the plane section. The coefficient of variation, showing the heterogeneity of the structure, is much smaller for the $3 \mathrm{D}$ structure $(0.16)$ than for plane section $(0.42)$.

Table. The standard version parameters of the plane section of the grain structure with the previously known distribution $P\left(D_{k}\right)$, giving the distribution of plane sections $P_{k}(d)$, corresponding to the quantitative characteristics of the standard G6 of ISO 643:2012

\begin{tabular}{|c|c|c|}
\hline Analysis object & Structure parameters & $\begin{array}{c}\text { Values of } \\
\text { parameters }\end{array}$ \\
\hline $\begin{array}{c}\text { Distribution of } \\
\text { grain diameters, } \\
\qquad P\left(D_{k}\right)\end{array}$ & $\begin{array}{l}\text { Mean diameter of grains } D, \mu \mathrm{m} \\
\text { Mean square deviation from mean diameter, } \mu \mathrm{m} \\
\text { Coefficient of variation of the grain diameters distribution } \\
\text { Modal diameter of grains, } \mu \mathrm{m} \\
\text { Maximum diameter of grains, } \mu \mathrm{m} \\
\text { Number of grains per } 1 \mathrm{~mm}^{3}, \mathrm{pc} \text {. }\end{array}$ & $\begin{array}{l}72.3 \\
10.7 \\
0.14 \\
73.3 \\
97.8 \\
5060\end{array}$ \\
\hline $\begin{array}{c}\text { Distribution of } \\
\text { plane sections } \\
\text { diameters, } \\
P_{k}(d)\end{array}$ & $\begin{array}{l}\text { Mean diameter of plane grains } d, \mu \mathrm{m} \\
\text { Mean square deviation from mean diameter, } \mu \mathrm{m} \\
\text { Coefficient of variation of the diameter distribution } \\
\text { Modal diameter, } \mu \mathrm{m} \\
\text { Mean area of plane grain } a, \mu \mathrm{m}^{2} \\
\text { Maximum diameter of plane grains, } \mu \mathrm{m} \\
\text { Number of plain grains } m \text { per } 1 \mu \mathrm{m}^{2}, \mathrm{pc} \text {. }\end{array}$ & $\begin{array}{r}44.2 \\
20.3 \\
0.46 \\
58.7 \\
1953 \\
97.8 \\
512 \\
\end{array}$ \\
\hline $\begin{array}{c}\text { Chords length } \\
\text { distribution, } \\
P_{k}(l)\end{array}$ & $\begin{array}{l}\text { Mean chord } l, \mu \mathrm{m} \\
\text { Mean square deviation from mean chord, } \mu \mathrm{m} \\
\text { Coefficient of variation of the distribution of chords } \\
\text { Modal size chord, } \mu \mathrm{m} \\
\text { Number of chords per } 1 \mu \mathrm{m}, \mathrm{pc} \text {. }\end{array}$ & $\begin{array}{c}39.0 \\
18.6 \\
0.48 \\
44.0 \\
26\end{array}$ \\
\hline $\begin{array}{c}\text { Structure } \\
\text { parameters of the } \\
\text { standard } G 6 \text { of } \\
\text { ISO 643:2012 }\end{array}$ & $\begin{array}{l}\text { Mean diameter of plane grains } d, \mu \mathrm{m} \\
\text { Nominal number of plane grains } m \text { per } 1 \mu \mathrm{m}^{2}, \mathrm{pc} \text {. } \\
\text { Mean area of plane grain } a, \mu \mathrm{m}^{2} \\
\text { Mean chord } l, \mu \mathrm{m} \\
\text { Maximum diameter of the plane grains, } \mu \mathrm{m} \\
\text { Number of chords per } 1 \mu \mathrm{m}, \mathrm{pc} \text {. }\end{array}$ & $\begin{array}{c}44.2 \\
512 \\
1950 \\
40.0 \\
97.8 \\
25\end{array}$ \\
\hline
\end{tabular}

After determining the distribution of $P_{k}(d)$, you can create the standard in the form of a grid, as is done in ISO 643:2012. This study showed that it is expedient to create standards of plane sections of 3D structures using the presented method.

\section{Conclusions}

The presented stereological method is applicable both for polyhedral structures and for structures with spherical particles. To take into account the shape of grain-polyhedron, the resulting equations of the method introduce the shape factors. An original method for determination of the shape factors is proposed, which can be applied to other polyhedral structures. The method can be used for creation standards of plane sections of 3D structure with a previously known size distribution $P\left(D_{k}\right)$ of $3 \mathrm{D}$ grains or other particles.

\section{References}

1. S. Berbenni, V. Favier, M. Berveiller. Int J Plast, 23, 114 - 142 (2007) 
2. T. Wejrzanowski, S. H. Ibrahimb, J. Skibinski, K. Cwieka, K. I. Kurzydlowski. Image Anal Stereol, 36, 105-110 (2017)

3. B. Biswal, P-E Øren, R. J. Held, S. Bakke, R. Hilfer. Image Anal Stereol 28, 23-34 (2009)

4. G. Liu, H. Yu. Image Anal Stereol 19, 81-84

5. L. Bizet, J. Breard, G. Bouquet, J.-P. Jernot, M. Gomina. Image Anal Stereol 23, $167-$ 176 (2004)

6. A. Tewari, A. M. Gokhale. Mat Char 46, 329-335 (2001)

7. H. E. Exner. Image Anal Stereol 23, 73-82 (2004)

8. L. M. Cruz-Orive (2017). Image Anal Stereol 36, 153-177 (2017)

9. S. A. Saltykov. Stereometric metallography (Moscow, Metallurgy, 1970)

10. E. E. Underwood. Quantitative stereology (Reading MA:Addison-Wesley, 1970).

11. C. Germain, R. Blanc, M. Donias, O. Lavialle, J.-P. Da Costa, P. Baylou. Image Anal Stereol 24, 127-134 (2005)

12. H. He, Z. Guo, P. Stroeven, M. Stroeven, L. J. Sluys. Image Anal Stereol 29,79-84 (2010)

13. H. E. Exner. Acta Stereol 6/III, 1023-1028 (1987)

14. T. P. Danilenko. Proc 1-st Int Conf on Devel Test Appl Mat. Oct 2-5; Opatija, Croatia. 89-95 (1996)

15. T. P. Danilenko. Metallurgical and Mining Industry 4, 82-88 (2016)

16. T. P. Danilenko, E. I. Danilenko. Patent for invention No 113112 Ukraine. Prom vlasnist 23, 3, 64-65 (2016)

17. D. Stoyan. Image Anal Stereol 33, 225-229 (2014)

18. J. C. Tucker, L. H. Chan, G. S. Rohrer, M. A. Groeberc, A. D. Rollett. Scripta Mater 66, 554-557 (2012)

19. H. Adrian. Proc 3-d Conf on Stereol in Mat Science. Oct; Krakow, Poland. 131-142 (1990)

20. F. C. Hull FC, W. G. Houk WG. J of Met. April, 565-572 (1953)

21. K. Matsuura, Y. Itoh. Mater Trans, JIM 32/11, 1042-1047 (1991)

22. T. P. Danilenko. Metaloznavstvo ta obrobka metaliv 3, 35-42 (2010)

23. BS EN ISO 643:2012 Steels. Micrographic determination of the apparent grain size 